\title{
Research of fiber optical faceplate defects segmentation based on improved watershed algorithm
}

Bingqian Yang, Mingquan Wang, Junsheng Zhang, Jinkai Gao

Bingqian Yang, Mingquan Wang, Junsheng Zhang, Jinkai Gao, "Research of fiber optical faceplate defects segmentation based on improved watershed algorithm," Proc. SPIE 10452, 14th Conference on Education and Training in Optics and Photonics: ETOP 2017, 104526H (16 August 2017); doi:

10.1117/12.2269951

Event: 14th Conference on Education and Training in Optics and Photonics, ETOP 2017, 2017, Hangzhou, China 


\title{
Research of fiber optical face plate defects segmentation based on improved watershed algorithm
}

\author{
Yang Bingqian ${ }^{1,2}$,Wang Mingquan ${ }^{1,2}$,Zhang Junsheng ${ }^{1,2}$,Gao Jinkai ${ }^{1,2}$ \\ (1.Key Laboratory of Instrumentation Science \& Dynamic Measurement,North University of \\ China,Taiyuan030051,china 2.School of Information and communication Engineering,North \\ University of China,aiyuan03005 1,China)
}

\begin{abstract}
In this paper, an improved adaptive watershed segmentation method is proposed based on the characteristics of the optical fiber faceplate Firstly, median filtering and morphological contrast enhancement are performed on the defect images, and then the gradient of the image is obtained by multi-scale morphology.In the improved watershed algorithm, the local minimum is first removed which the depth is lower than H.Then, the local minimum of the depth larger than $\mathrm{H}$ as the seed point are extended.Finally,the gradient image is modified by the forced minimum method .The modified gradient image is used to make the watershed Segmentation to get the final segmentation result .The experimental results show that the method can effectively suppress the over-segmentation, and the defects can be extracted well.
\end{abstract}

Key words: image segmentation; watershed; adaptive market; optical fiber faceplate;

\section{INTRODUCTION}

In the manufacture of fiber optic panels, Process parameters fluctuate slightly or affect by other external factors Regularly arranged optical fibers are pressed against each other,Causing that some local area of the fiber can not transmit light or transmittance greatly reduced,producing defective and scrap products ${ }^{[1]}$.At present ,the Fiber Optical Faceplate of the automatic detection of defects indomestic research is very limited.However,The image segmentation as an important stage, the segmentation results directly affect the results of the detection.So it is very important to make a good segmentation result ${ }^{[2]}$.At present, there are many techniques of image segmentation, which can be divided into basic segmentation technology and advanced segmentation technology. ${ }^{[3]}$ The basic segmentation technology mainly has the threshold segmentation based on histogram and the edge detection technology based on the differential operator

*Wang mingquan;wangmq@nuc.edu.cn; phone 13934548995; Yang bingqian;157736354@qq.com;phone 18331235503; Zhang junsheng;45993095@qq.com;phone 13835143630;

14th Conference on Education and Training in Optics and Photonics: ETOP 2017, edited by Xu Liu, Xi-Cheng Zhang, Proc. of SPIE Vol. 10452, 104526H · C 2017 ICO, IEEE, OSA, SPIE

CCC code: $0277-786 X / 17 / \$ 18 \cdot$ doi: $10.1117 / 12.2269951$

Proc. of SPIE Vol. $10452104526 \mathrm{H}-1$ 
Advanced segmentation techniques are based on the segmentation technique combined with the theory of certain fields(E.g mathematical morphology ${ }^{[4]}$ neural network Statistical pattern recognition ${ }^{[5]}$ and so on).In the field of image segmentation, there is no general segmentation method because of the complexity of the image. In many segmentation methods, The segmentation method based on watershed theory is intuitive $[$, fast and always produces a complete boundary ${ }^{[6]}$,Having a good response to the weak edge .It provides the possibility to obtain the closed edge of the defect image ${ }^{[7]}$. Based on the above advantages .we use the improved watershed segmentation algorithm to segment the defect of the optical fiber face plate.Experimental results show that The method of this paper has a good detection effect on the defects of uneven illumination, low contrast and small area.Improve the effect and efficiency of the detection of the optical fiber Faceplate.

\section{WATERSHED SEGMENTATION METHOD AND ITS SHORTCOMINGS}

Watershed ${ }^{[8]}$ is a classical concept in geomorphology,In the field of image processing, Gray images can be viewed as terrain surface, The gray value of each pixel in the image represents the height of the point,Every local minimum area and its influence called the catchment basin, stabbing a hole in the bottom of each area, Water from the bottom begin to swell,and slowly drowned the entire area of the image when the water from different bottom is converging, build a hypothetical dam to stop it. When the immersion process is over, each area is flooded and completely surrounded by the dam. These built dams is the watershed of the area ,that is, the outline of the image. This method is not only fast, shorten the execution time, and the results are more accurate, with high practical value.But the shortcomings of the traditional watershed algorithm is over segmentation, That is, the image is divided into too many small areas, and the target of interest is submerged. In order to eliminate the over segmentation of the watershed algorithm, it can usually be improved from three aspects.(1)Preprocessing filter(2)sign(3)Region merging.In this paper, based on the characteristics of the surface defects of the optical fiber panel ,A new algorithm for defect segmentation of optical fiber face plate is presented from the aspects of preprocessing filter and marking.

\section{WATERSHED SEGMENTATION ALGORITHM BASED ON ADAPTIVE MARKER}

\subsection{Image preprocessing}

In view of the traditional watershed segmentation algorithm is sensitive to noise, easy to produce over segmentation.Filtering is performed before the watershed segmentation to reduced the impact of pseudo local minimum on the segmentation results. The median filter is a nonlinear signal processing method, which has a certain protective effect on the edge.the median filter with a window size of $3 * 3$ is used to smooth the image to eliminate the influence of noise while suppressing the number of local pseudo-minimums.

\subsection{Contrast enhancement}

After median filtering, the image noise can be suppressed, but the edge of the small defect becomes weak.In mathematical morphology, The high-hat ${ }^{[9]}$ transform is defined as the difference between the original image and the open operation. The high-hat transform has some properties of high-pass filtering, emphasizing the gray-scale peak of the object, enhancing the edge information of the image.Its definition is as follows:

$$
T_{\text {hat }}(f)=f-(f \circ b)
$$


$\mathrm{f}$ is a gray image, $\mathrm{b}$ is a structural element.

The bottom-hat ${ }^{[10]}$ transform is defined as the difference between the closed operation of the image and the original image.The bottom-hat transform can find the valley in the image and highlight the boundary between the connected objects.Its definition is as follows:

$$
B_{\text {hat }}(f)=(f \bullet b)-f
$$

The combination of high-hat transform and bottom-hat transform can make the image foreground and background gray level be stretched, highlight the relevant targets and details, and play the role of image enhancement.we use difference between high-hat and bottom-hat transform to realize image contrast enhancement.the structural elements selected $4 *$ 4 size of the circular structure.Its expression is as follows :

$$
G=f+T_{\text {hat }}(f)-B_{\text {hat }}(f)
$$

$\mathrm{f}$ is a gray image, $\mathrm{b}$ is a structural element, $\oplus$ is Morphological dilation operation , $\Theta i s$ morphological erosion operation.

\subsection{Multi-scale morphological gradient}

The gradient image is usually used as the direct object to deal with the watershed transform, which can well reflect the change of the gray level of the image pixel.We want to extract the edge of the target is often the gradient of the obvious changes in the region. Therefore, the watershed segmentation of the gradient image can be better than the original image segmentation.

The traditional morphological gradient operator is as follows :

$$
G(f)=(f \oplus B)-(f \Theta B)
$$

$\mathrm{f}$ is a gray image, $\mathrm{b}$ is a structural element, $\oplus$ is Morphological dilation operation, $\Theta$ is morphological erosion operation.

The traditional morphological gradient use a single structural element to obtain gradient image. When the selected structural elements are over an hour, the details of the image are protected, but the ability to eliminate noise is weak. When the selection of the structural elements is too large, can remove most of the noise, but also damage the image of the details of the information, and ultimately lead to the defect can not be completely separated out .In view of the above characteristics, the paper uses the multi-scale morphological gradient operator to calculate the gradient of the image.The mathematical expression of the multi-scale gradient operator is as follows ${ }^{[1]]}$ :

$$
M G(\mathrm{f})=\frac{1}{n} \sum_{i=1}^{n}\left[\left(f \oplus B_{i}\right)-\left(f \Theta B_{i}\right) \Theta B_{i-1}\right]
$$

$\mathrm{N}$ is the scale factor,Structural element $\mathrm{Bi}$ is expanded Itimesby $\mathrm{B} 0, \mathrm{Bi}=\mathrm{B}_{0} \oplus \mathrm{B}_{0} \oplus \mathrm{B}_{0} \oplus \ldots \oplus \mathrm{B}_{0}{ }^{[12]}$ 。 After many simulation experiments, it is concluded that the circular structure elements with a diameter of 4 are selected in the multi-scale morphological gradient, and the size of the $n=2$ is suitable for the defect segmentation of the optical fiber Faceplate. 


\subsection{Adaptive marker extraction}

After more than two steps to obtain the gradient image, there are still a lot of pseudo area minimum .Directly to the gradient image watershed segmentation, there will be a serious over segmentation phenomenon.In order to further eliminate the local pseudo-minimum region, the local minimum region only appears in the target region.In this paper, an improved method of adaptive label extraction is propose.First, all the local minimums of the image are compared with $\mathrm{H}$,Mark local minimum with depth less than $\mathrm{H}$ as 0 and 1 with depth greater than $\mathrm{H}$,Eliminate the local minimum where the depth is less than $\mathrm{H}$, Next, the remaining local minimum region is expanded as a seed point, And all the points within 8 neighborhoods which are less than $\mathrm{H}$ in the seed point are included in the seed region. The local minimum value region generated by this method is used as the mark image, and the gradient image is modified by the method of the minimum imposition. The modified gradient image is divided into watershed segmentation. The selection of threshold $\mathrm{H}$ is the key point in this method.If $\mathrm{H}$ is chosen too large, the edges of the details of the image become blurred, resulting in defective extraction is incomplete.If $\mathrm{H}$ is chosen too small, the pseudo-local minimum can not be well suppressed, and over-segmentation is easy to occur ${ }^{[11]}$.Aiming at this feature, a large number of simulation experiments were carried out on the defect image of the fiber optic Face plate In this paper, we adopt the adaptive method to select the parameter H.The formula is as follows:

$$
H=\frac{M+S}{2}+S_{\max }+S_{\min }
$$

\section{EXPERIMENTAL RESULTS AND ANALYSIS}

\subsection{Analysis of important parameters of the algorithm}

This algorithm mainly involves the choice of two parameters, The Selection of Median Filter Window Size and The Selection of Morphological Structure Elements.In this paper, the effect of the segmentation results of the two parameters is illustrated by the example of the spot defect.

(1)Effect of median filter window size. Median filtering can filter out some noise, but if the window is too large, it is the edge of the defect becomes blurred, some small defects can not be identified.If the window is too small, it can not effectively reduce the influence of the local minimum.Figure 1 shows the effect of different filtering windows on the segmentation results.Fig. 1 (b) can be seen that the filter window is too small can not effectively suppress the local pseudo-minimum value resulting in over-segmentation.From Fig. 1 (d), it can be seen that the middle filter window is too large. Although the noise and pseudo local minimum are suppressed, some of the smaller defects are not segmented .After a large number of experiments, we use $3 * 3$ filter operator to filter the spot image. 


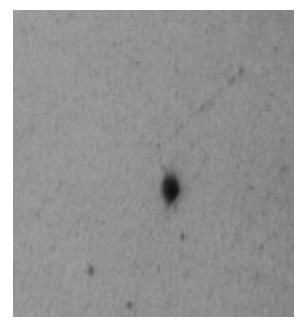

(a)original image

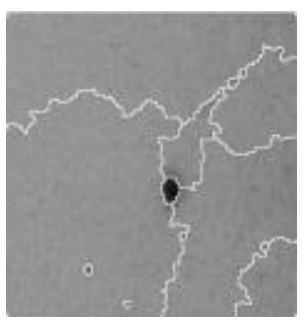

(b) $2 * 2$ Filter segmentation results

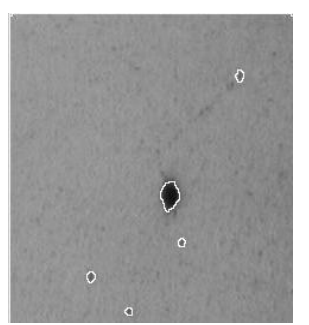

c)3*3Filter segmentation results

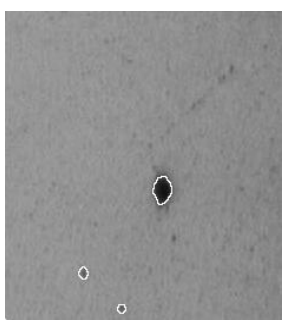

(d) $5 * 5$ Filter segmentation results

Fig.1Influence of Different Size Filter Operators on Segmentation

(2)Morphological structure elements

First of all, in order to maintain good isotropic should select the circular structure elements. Structural element is too large or too small can not be very good to achieve the final defective element segmentation.Fig.2 (b) shows the result of segmentation using $2 * 2$ size structural elements.Fig.2 (c) shows the segmentation result of $8 * 8$ structure elements. The background image is clearly misinterpreted as a defect in both images.

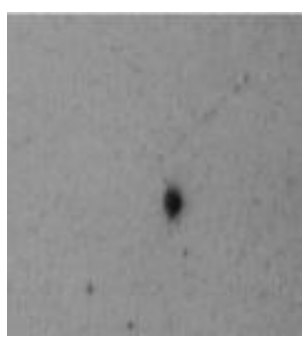

(a)original image

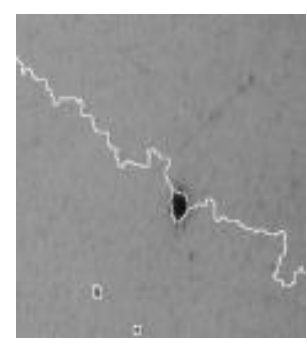

(b)results of $2 * 2$ Structural element

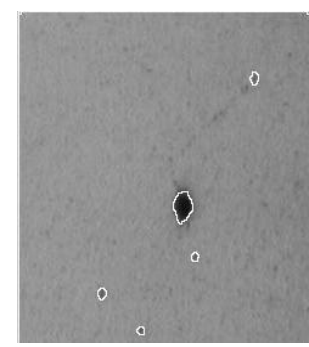

(c)results of $4 * 4$ Structural element

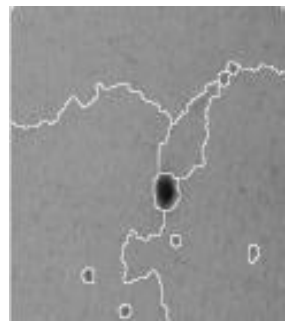

(b)results of $8 * 8$ Structural element

Fig.2 The Effect of Different Size Structural Elements on Segmentation

\subsection{Algorithm validity detection}

In order to verify the effectiveness of the algorithm, This paper uses the CPU for the Intel Celeron2.60GHz, memory 4GB computer as a hardware platform, Fiber panel spot and chickenwire image were used as test images, In the MATLAB2014a programming environment to achieve the above algorithm .The segmentation process shown in Fig. 3.Fig. 3 (a) is the local minimum position of original image after contrast enhanced we can see that a large number of local minimum appear in the background,Fig. 3 (b) is the traditional watershed segmentation results, we can see a large number of over segmentation phenomenon,Fig. 3(c) is the local minimum marked by this algorithm, we can find that the local minimum local only appeared in the target defect, a large number of local pseudo-minimum is removed,Fig. 3 (d) is the segmentation result of the algorithm in this paper, and the defects are basically segmented. 


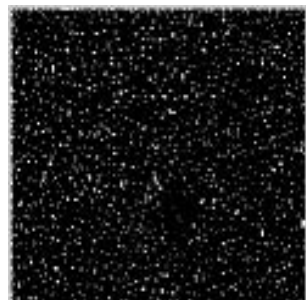

(a)local minimum region before modification

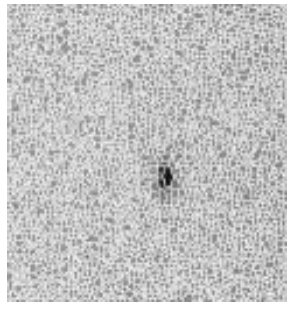

(b)the Segment result

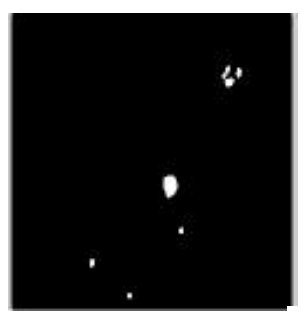

(c)Modified local minima

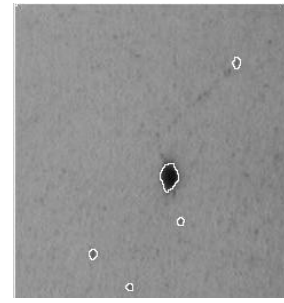

(d)Improved segmentation results

Fig.3Comparison the results of the spot segmentation

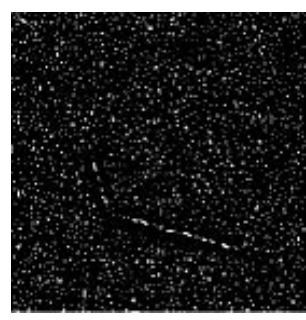

(a)local minimum region before modification

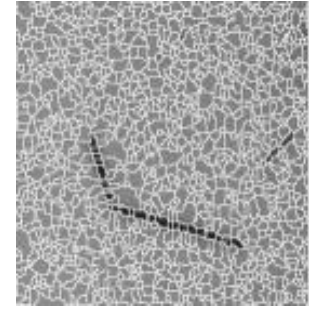

(b)the Segment result

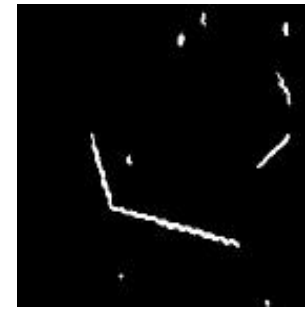

(c)Modified local minima

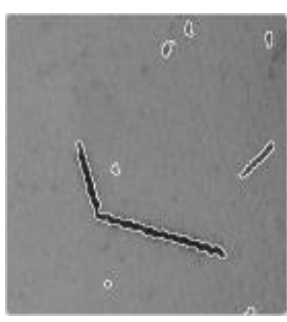

(d)Improved segmentation results

Fig.4Comparison the results of the chickenwire segmentation

\section{SUMMARY}

In this paper, an adaptive watershed segmentation method is proposed, which can effectively avoid the problem of over-segmentation of traditional watershed method.The experimental results show that the proposed method is suitable for the segmentation of defect images in fiber optic Faceplate, Which effectively solves the problem of over-segmentation in watershed segmentation and can produce better segmentation results.Can be used in machine vision based optical fiber Faceplate, defect detection system.

\section{REFERENCES}

[1] Edmund Industrial Optics. Electronic Imaging Resource Guide: report of Edmund Industrial Optics. Barrington: Edmund Industrial Optics, 2001.

[2] Okanai, F; Sakurai, H;etc. Characteristics of optical imaging capillary plate gas detector with fiber optic plate[J]. Nuclear Instruments \& Methods in Physics Research. Section A, Accelerators, Spectrometers, Detectors and Associated Equipment. 2007, 571(1-2):289-293.

[3] Masaru NISHIGUCH, Michisato TOYODA,etc. Development of a Miniature Double Focusing Mass Spectrograph Using a Focal Plane Detector[J]. Journal of the Mass Spectrometry Society of Japan.2006,54(1):1-9.

[4] V. Grau, A.U.J. Mewes, M. Alcaniz, R. Kikinis, S.K.Warfield, "Improved watershed transform for medical 
imagesegmentation using prior information”, IEEE Transactions onMedical Imaging, Vol.23(4), 2004, pp. 447-458

[5] L. Vincent, P. Soille, "Watersheds in digital spaces: anefficient algorithm based on immersion simulations", IEEETransactions on Pattern Analysis and Machine Intelligence,Vol. 13(6), pp. 583-59

[6] C.W. Chen, J. Luo, K.J. Parker, "Image segmentationvia adaptive K-mean clustering and knowledge basedmorphological operations with biomedical applications",IEEE Transactions on Image Processing, Vol.7 (12), 1998,pp 1673-1683

[7]. Indah Soesanti, Adhi Susanto, Thomas Sri Widodo and Maesadji Tjokronagoro, 2011. "Optimized FuzzyLogic Application For MRI Brain ImagesSegmentation”, International Journal of ComputerScience and Information Technology (IJCSIT) Vol 3, No 5, Oct 2011, pp: 137-146.

[8] Herman van Dyk; Perry Peralta, Development of a Wood Fiber Panel Product Containing Polypropylene/Polyester Bicomponent Fiber[C], International Conference on Woodfiber-Plastic Composites(and other natural fibers); 20050523-25; Madison,WI(US),2007, P.435.

[9]Lakshmi, S. and V. Sankaranarayanan, 2010. "A Study of edge detection techniques for segmentation computing approaches", .Computer Aided Soft Computing Techniques for Imaging and Biomedical Applications, pp: 35-41

[10] Ji, Y., J. Zhang, J. Meng, X. Zhang, 2010, “A new CFAR shiptarget detection method in SAR Imagery”, Acta Oceanol. Sin.,Vol.29, No.1, pp.12-16.

[11] Longadge, R., S. S. Dongre, L. Malik, "Class ImbalanceProblem in Data Mining: Review”, Int. J. Comp. Sci. andNetw., Vol. 2, Iss. 1, Feb. 2013.

[12] Brekke, C. , A. H.S. Solberg , 2005, "Oil spill detection bysatellite remote sensing", Remote Sensing of Environment, Vol.95, pp.1-13. 